\title{
Análisis Presupuestal de Gestión de Riesgos de Desastres en salud, Lima-Norte ${ }^{1}$
}

\section{Budget Analysis of Health Disaster Risk Management, Lima-Norte}

\author{
Alberto Gonzales Guzmán ${ }^{2}$ \\ Universidad Peruana Cayetano Heredia, Lima, Perú \\ gonguz.beto@gmail.com
}

Recibido: 3/09/2020 - Aceptado:26/06/2021 - Publicado: 06/12/2021

\section{RESUMEN}

Determinar la relación de las metas presupuestales con las metas físicas del Programa presupuestal 068 "Reducción de la Vulnerabilidad y Atención de Emergencias por Desastres" de la Dirección de Redes Integradas de Salud Lima Norte (DIRIS Lima Norte), 2019. Metodología: Enfoque cuantitativo; la población estuvo conformada por todos los ítems mediante los cuales se dividió el presupuesto inicial modificado, a modo de una muestra censal. Se usó el método hipotético-deductivo y se aplicó la prueba de Wilcoxon. Resultados: Con un nivel de significancia de 5\%, se obtuvo un p-valor $>0,05$, por lo que, se afirma que en el análisis presupuestal no se encuentra una relación entre la ejecución de las metas presupuestadas y las metas físicas de la DIRIS Lima Norte, mediante lo cual puede establecerse dos situaciones: (a) baja ejecución presupuestal con alta ejecución de metas físicas; o (b) alta ejecución presupuestal con baja ejecución de metas físicas. Conclusiones: Las metas presupuestales no se relacionan con las metas físicas de la DIRIS Lima Norte.

Palabras clave: análisis presupuestal; gestión de riesgo de desastres en salud; DIRIS Lima-Norte.

Código JEL: H5 National Government Expenditures and Related Policies; H51 Government Expenditures and Health.

(C) Los autores. Este artículo es publicado por Pensamiento Crítico de la Facultad de Ciencias Económicas, Universidad Nacional Mayor de San Marcos. Este es un artículo de acceso abierto, distribuido bajo los términos de la licencia Creative Commons Atribucion - No Comercia_Compartir Igual 4.0 Internacional. (http://creativecommons.org/licenses/by-nc-sa/4.0/) que permite el uso no comercial, distribución y reproducción en cualquier medio, siempre que la obra original sea debidamente citada. 


\section{ABSTRACT}

To determine the relationship of the budget goals with the physical goals of the Budget Program 068 "Vulnerability Reduction and Disaster Emergency Response" of the Directorate of Integrated Health Networks North Lima (DIRIS North Lima), 2019. Methodology: Quantitative approach; the population was made up of all the items by which the modified initial budget was divided, as a census sample. The hypothetic-deductive method was used and the Wilcoxon test was applied. Results: With a significance level of $5 \%$, a p-value $>0.05$ was obtained, therefore, it is stated that in the DIRIS North Lima budget analysis there is no relationship between the execution of the budgeted goals and the physical goals, through which two situations can be established: (a) low budget execution with high execution of physical goals; or (b) high budget execution with low execution of physical goals. Conclusions: The budget goals are not related to the physical goals of DIRIS Lima-Norte. Keywords: budget analysis; health disaster risk management; DIRIS North Lima. Código JEL: H5 National Government Expenditures and Related Policies; H51 Government Expenditures and Health. 


\section{Introducción}

En esta sección se describe las consideraciones generales sobre la jurisdicción sanitario de análisis, es decir, la Dirección de Redes Integradas de Salud Lima Norte, y las características territoriales de riesgo de desastres, a fin de poder situar el escenario de desarrollo de la presente investigación.

\subsection{La Dirección de Redes Integradas de Salud Lima Norte}

La capital del Perú es Lima Metropolitana, la cual posee 43 distritos. Lima Metropolitana se divide en 04 jurisdicciones desde el enfoque de gestión en salud: Centro, Norte, Este y Sur, cada una responsable por una Dirección de Redes Integradas de Salud que aglomera diferentes cantidades de distritos según criterios de articulación y accesibilidad de la población. La Dirección de Redes Integradas de Salud Lima Norte, cuya abreviatura es DIRIS Lima Norte, es un órgano desconcentrado del MINSA (Ministerio de Salud, del Perú), cuya responsabilidad recae en la operación, gestión y articulación de procesos para promocionar, prevenir, recuperar y rehabilitar en Salud, constituyendo la Red Integrada de Salud, que conforma hospitales y establecimientos de salud del primer nivel de atención, en el cual forman parte nueve distritos de Lima Metropolitana en la zona norte, como: Ancón, Carabayllo, Comas, Independencia, Los Olivos, Puente Piedra, Rímac, San Martín de Porres y Santa Rosa. La misión de la DIRIS Lima Norte es otorgar garantía para otorgar oportuna y adecuadamente las prestaciones de salud a la población, realizar el desarrollo de acciones relacionadas con intervenciones sanitarias de salud pública, respuesta a las emergencias/desastres, generando el valor de docencia e investigación; con una visión de conformarse como una entidad pública líder en la Atención Integral de Salud (DIRIS Lima Norte, 2020).

La estructura organizacional de la DIRIS Lima Norte fue aprobada mediante la Resolución Ministerial N 467-2017/MINSA. Se evidencia en el núcleo operativo tres órganos de línea: Dirección de Monitoreo y Gestión Sanitaria, Dirección de Medicamentos, Insumos y Drogas y la Dirección de Salud Ambiental e Inocuidad Alimentaria (DIRIS Lima Norte, 2020).

La Dirección de Monitoreo y Gestión Sanitaria, que es un órgano de línea de la DIRIS Lima Norte, subordina a la Oficina de Gestión de Riesgo 
de Desastres, la cual compone cuatro equipos funcionales de trabajo: (a) espacio de monitoreo de emergencia y desastres; (b) capacitación y doctrina; (c) planeamiento; y, (d) movilización y logística en gestión de riesgos y desastres (DIRIS Lima Norte, 2020).

La DIRIS Lima Norte subordina a 104 establecimientos de salud (ES), 3 servicios médicos de apoyo (SMA), un 1 instituto (Instituto Nacional de Salud Mental "Honorio Delgado - Hideyo Noguchi y 3 hospitales, los cuales son (a) el Hospital Nacional Cayetano Heredia; (b) el Hospital Nacional Sergio Bernales; y (c) el Hospital Carlos Lanfranco la Hoz. También subordina, ya que es la autoridad sanitaria en 9 distritos, a los ES, SMA y hospitales de otros subsectores, como: (a) Essalud, (b) las fuerzas armadas (policía, ejército, aviación y marina), (c) las municipalidades y (d) instituciones del sector privado (DIRIS Lima Norte, 2020).

\subsection{Gestión del Riesgo de Desastres}

En la República del Perú existen condiciones geográficas que nos hacen vulnerables ante sismos; los deslizamientos; los derrumbes; las erosiones; las inundaciones; las variaciones climáticas que afectan determinadas regiones, como las precipitaciones, los vientos intensos, las granizadas y las heladas, entre otros (Kuroiwa, 2010; Pontificia Universidad Católica del Perú, 2016).

El riesgo de desastres implica dos conceptos claves: (a) los peligros y (b) las vulnerabilidades. Los peligros consisten en la probabilidad de que fenómenos con potencial lesivo, sea de orden natural o antrópico, haga su aparición en un espacio geográfico específico, caracterizado por una determinado poder de daño (intensidad), duración y repetición en el tiempo de modo definido, cuya clasificación puede observarse en la figura 1. Las vulnerabilidades consisten en aquella susceptibilidad que tiene la población, la estructura física o la actividad socioeconómica para padecer der daños por la acción de un peligro o una amenaza; teniendo como factores: (a) la exposición, (b) la fragilidad y (c) la resiliencia, de la población y de sus medios de vida (INDECI, s/f; El Peruano, 2011; Ministerio del Ambiente, 2011; Dirección General de Cambio Climático y Desertificación, 2018; Ministerio del Ambiente, 2018). 
La Gestión de Riesgo de Desastres (GRD) consiste en el análisis y la intervención de factores que generan riesgos basados en la cercana correlación entre los conceptos de amenaza, peligro, y vulnerabilidad, toda vez que están interconectados y en un constante dinámica de cambio afectadas por el ser humano en el territorio y también por la variabilidad climática agudizada en la actualidad por el cambio climático. Por lo que, el riesgo y sus factores son cambiantes antes, durante y después de los desastres (Programa Nuestras Ciudades, 2014).

\section{Marco Teórico}

En esta sección se desarrolla el marco teórico-normativo desde el enfoque económico de la Gestión del Riesgo de Desastres y la operatividad a nivel nacional considerando las leyes, reglamentos y otros documentos normativos que detallan el enfoque y procedimientos a seguir en el campo de la salud.

\subsection{Estrategia de Gestión Financiera del Riesgo del Desastre en el Perú}

Según la Ley Nº 29664 (El Peruano, 2011), existe la Estrategia de Gestión Financiera del Riesgo del Desastre, instrumento del Sistema Nacional de Gestión del Riesgo de Desastres que consiste en el grupo de intervenciones para asegurar una adecuada capacidad financiera en los procesos de la GRD y que permita brindar una cobertura ante los riesgos fiscales que ha de derivarse por los efectos del desastre; para ello se desarrollaron los siguientes mecanismos: (a) el Presupuesto por Resultados; y (b) la protección financiera. El presupuesto por resultado tiene como función principal la reducción de las vulnerabilidades que incrementan las condiciones de riesgo y el desarrollo de capacidades para dar respuesta una vez sucedidos los desastres; también ejercer una función de priorización de la gestión prospectiva y gestión correctiva del riesgo de desastres, así como del proceso de preparación para la gestión reactiva del riesgo, siendo el instrumento presupuestal ad hoc el Programa Presupuestal 068 "Reducción de la Vulnerabilidad y Atención de Emergencias por Desastres” (PP 068). La protección financiera tiene su valor primordial una vez ocurrido el desastre, para favorecer la puesta en operaciones de servicios; además, considera la reserva de 
contingencia para la atención de emergencias (ver tabla $N^{\circ} 01$ ) (Programa Nuestras Ciudades, 2014).

Tabla $\mathbf{N}^{\circ} 01$

Mecanismos de gestión financiera del riesgo del desastre.

\begin{tabular}{ll}
\hline Estrategia de Gestión Financiera del Riesgo del Desastre \\
\hline Presupuesto por Resultados & Programas Presupuestales: 068, 048, 061, 089, 104 y 0111. \\
& Plan de Incentivos a la Mejora de la Gestión y Modernización Municipal. \\
& Fondos de Transferencias: FONIPREL y FONIE. \\
\hline Protección Financiera & Reserva de Contingencia a favor del INDECI. \\
& Líneas de Crédito Contingentes (ex ante). \\
& Préstamos de la Cooperación Bilateral o Multilateral (ex post). \\
& Fondo de Estabilización Fiscal. \\
& Transferencia del Riesgo (seguros y reaseguros, instrumentos paramétricos y \\
& otros instrumentos del mercado). \\
\hline
\end{tabular}

Fuente: Programa Nuestras Ciudades, 2014.

Cabe resaltar que existen varios tipos de peligros considerando su causa, los que devienen de: (a) geodinámica interna, como terremotos; (b) geodinámica externa; y (c) fenómenos oceanográficos e hidrometeorológicos (ver figura $\mathrm{N}^{\circ}$ 01) (PREVAED, 2020).

De estos tres tipos de peligros, se puede considerar las referencias que vienen a continuación. Las zonas sísmicas en Lima Norte de muy alto peligro y zona de riesgo crítico (nivel IV y V) comprenden los distritos de Ancón, de Puente Piedra y de Carabayllo (ver tabla $\mathrm{N}^{\circ} 02$ ). En Lima, la mayor susceptibilidad por precipitaciones pluviales se ubica en la geografía denominada "laderas de las cuencas medias y altas" de los ríos Chillón, Rímac (ambas en la DIRIS Liman Norte) y Lurín. Los puntos críticos ante inundaciones en Lima Norte, son los vinculados al recorrido del rio Rímac y Chillón, ámbitos, en los cuales se han caracterizado desbordes de cauces por fenómenos hidrometereológicos, que hicieron necesario la implementación de actividades permanentes de mantenimiento de las pendientes de equilibrio, descolmatación y construcción de obras para la corrección y control de cauce. El evento denominado "Fenómeno Niño Costero", más reciente, fue el del año 2017, se declararon en emergencia 15 distritos de Lima Metropolitana, ocasionándose episodios de lloviznas, crecidas e inundaciones de los ríos que atraviesan la ciudad de Lima, lo cual afectó no solo a las vías de comunicación, también a la salud de la población y a las mismas instalaciones de salud de la capital. Los distritos de Lima Norte declarados en emergencia 
fueron: Rímac, Carabayllo, Puente Piedra, Los Olivos, Comas y San Martín de Porres (PREVAED, 2020).

\section{Figura $\mathbf{N}^{\circ} 01$}

Clasificación de los peligros.

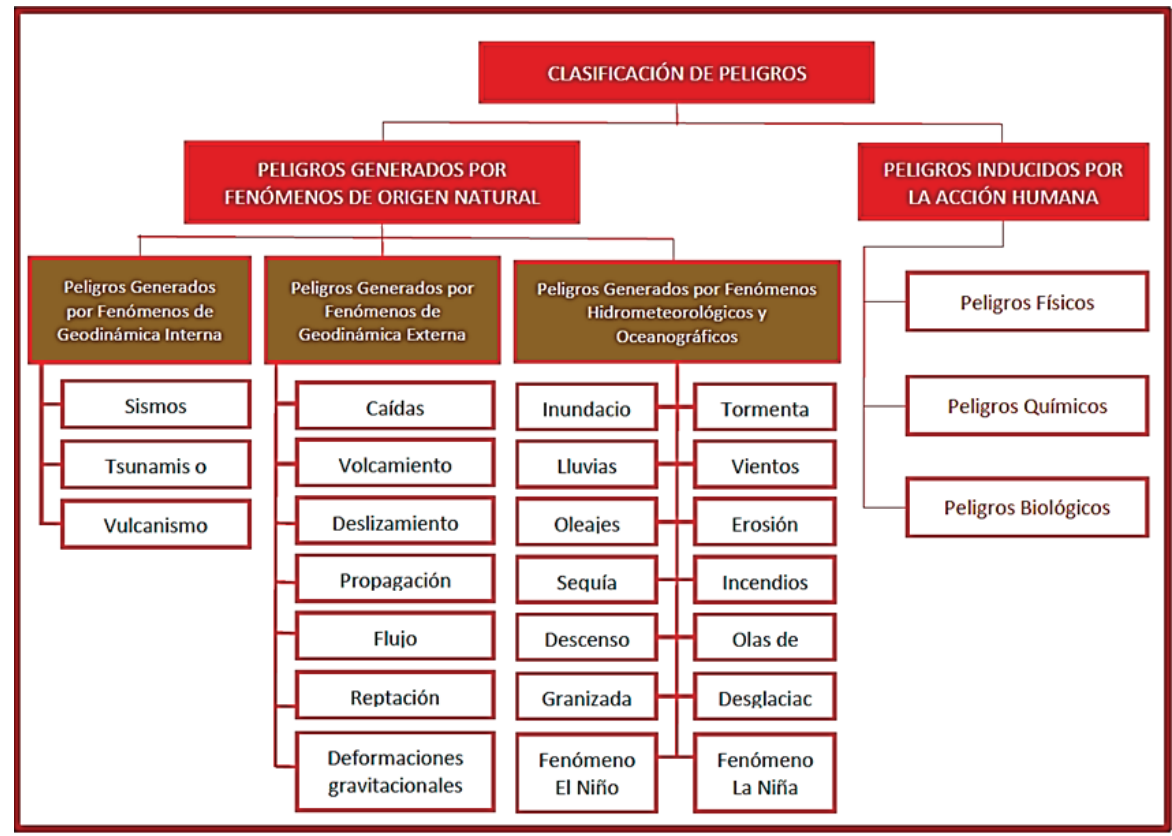

Fuente: PREVAED, 2020.

Tabla $\mathbf{N}^{\circ} 02$

Diseño de escenario sobre el impacto de un sismo de gran magnitud en Lima metropolitana y el Callao - distritos de Lima Norte, 2020.

\begin{tabular}{lllllll}
\hline \multirow{2}{*}{ Distrito } & Población & \multicolumn{2}{l}{ Niveles de vulnerabilidad } & & & \\
& (habitantes) & No afectada & Heridos & \% heridos & Fallecido & \% fallecidos \\
\hline Ancón & 48674 & 48139 & 487 & $1 \%$ & 48 & $0.1 \%$ \\
Carabayllo & 321752 & 256432 & 61137 & $19 \%$ & 4183 & $1.3 \%$ \\
Comas & 570735 & 454838 & 108475 & $19 \%$ & 7422 & $1.3 \%$ \\
Independencia & 235261 & 230795 & 4023 & $2 \%$ & 443 & $0.2 \%$ \\
Los Olivos & 401634 & 392929 & 7894 & $2 \%$ & 810 & $0.2 \%$ \\
Puente Piedra & 377993 & 301259 & 71820 & $19 \%$ & 4914 & $1.3 \%$ \\
Rímac & 181919 & 177826 & 3715 & $2 \%$ & 378 & $0.2 \%$ \\
San Martín de Porres & 751073 & 737043 & 12852 & $2 \%$ & 1178 & $0.2 \%$ \\
Santa Rosa & 20299 & 20076 & 203 & $1 \%$ & 20 & $0.1 \%$ \\
Total & 2909340 & 2619336 & 270606 & $9 \%$ & 19397 & $1 \%$ \\
\hline
\end{tabular}

Fuente: Elaboración propia. 


\subsection{El Programa Presupuestal 068 “Reducción de la vulnerabi- lidad y atención de emergencias por desastres"}

El Presupuesto por Resultados consiste en una intervención estratégica de gestión pública que sirve para asociar vinculantemente los recursos presupuestales para bienes y servicios en productos específicos que sean favorables a la población de manera cuantificable y directamente proporcional. Su propósito es contribuir con la mejora de la calidad del gasto público para que las entidades del Estado sean más eficaces y eficientes en el uso de los recursos públicos, dando prioridad en el gasto público de provisión de bienes y servicios que, en base a la evidencia, sirven para lograr resultados asociados al bienestar de la población y para que se tomen mejores decisiones en materia presupuestal y de gestión, para el logro de resultados de impacto en la población. Es implementado mediante cuatro instrumentos: (a) los programas presupuestales, (b) el seguimiento, (c) las evaluaciones independientes y (d) los incentivos a la gestión (Gob.pe, 2019).

Los programas presupuestales (PP) consisten en las unidades de programación de acciones de entidades públicas, las cuales al ser integradas y articuladas se enfocan en la provisión de bienes y servicios, para el logro de un resultado específico que favorezca a la población y de esta manera ejercer una contribución al logro de un resultado final relacionado con un objetivo de la política pública (Gob.pe, 2019).

El PP 068 es un programa presupuestal multisectorial, el cual está integrado por más de dos entidades del Gobierno Nacional para la provisión de servicios. El ente Rector del PP 068 es la Presidencia del Consejo de Ministros mediante el PREVAED. Este programa integra a entidades de distinto nivel de gobierno en el marco de sus competencias, de las cuales en el Gobierno Regional cuenta con 26 pliegos regionales y 1,070 municipalidades. La población objetivo del PP 068 es universal, es decir, para los más de 31 millones de habitantes del país (ver figura $N^{\circ} 02$ ) (PREVAED, 2019). 
Figura $\mathbf{N}^{\circ} 02$

Peligros priorizados por el PP 068 al año 2020.

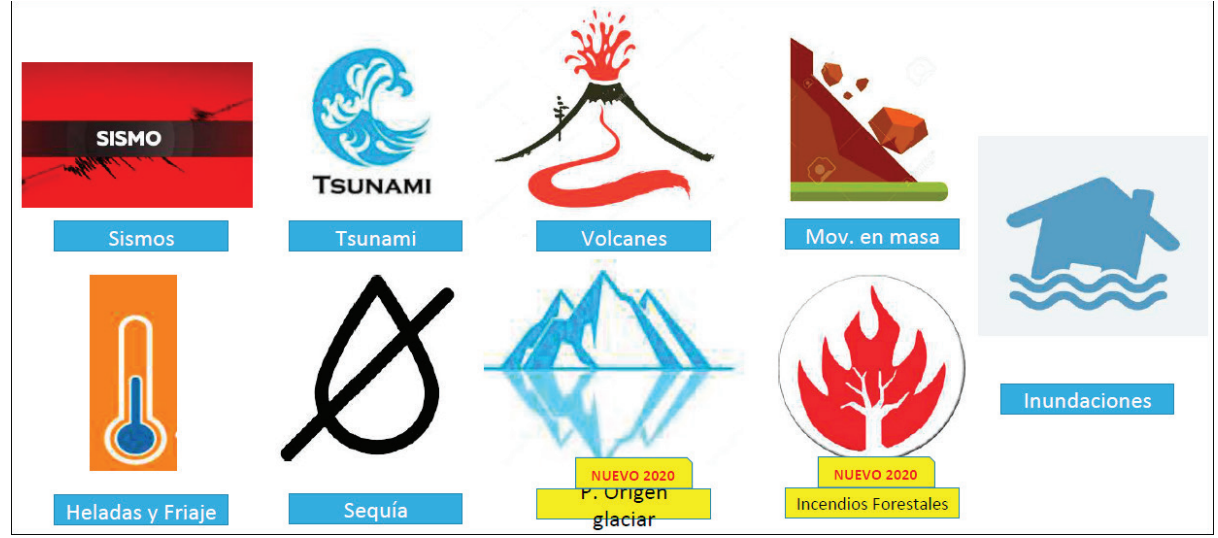

Fuente: PREVAED, 2019.

El Programa Presupuestal 068 "Reducción de la vulnerabilidad y atención de emergencias por desastres" se creó a través del Decreto de Urgencia № 024-2010 “Dictan medidas económicas y financieras para la creación del Programa Presupuestal Estratégico de Reducción de la Vulnerabilidad y Atención de Emergencias por Desastres en el Marco del Presupuesto por Resultados". El PP 068 como punto de partida identifica el problema específico con la afirmación de que la población y la infraestructura pública se encuentran bajo la exposición y vulnerabilidad de ocurrencia de peligros que se generan por fenómenos naturales o por acción humana. El PP 068 comprende intervenciones en gestión correctiva y reactiva, aunque pretende impulsar intensamente el componente de la gestión prospectiva, es decir, la prevención del riesgo, por medio de la formulación y difusión de mapas de peligros, así como el desarrollo de tecnologías para ser aplicadas hacia el entorno físico (PREVAED, 2020).

Desde el año 2010 hasta la fecha (2020) la forma como se organizan productos y actividades del PP 068 ha variado, debido a lo complejo de ser un programa presupuestal multisectorial, vinculado a varios ministerios, y para los tres niveles de gobierno: nacional, regional y local. La estructura funcional vigente relacionada con el sector salud se aprecia en la tabla $\mathrm{N}^{\circ} 03$, en donde se puede apreciar cinco productos, una acción común, 13 actividades y 21 finalidades. 
Tabla $\mathbf{N}^{\circ} 03$

Estructura funcional programática del PP 068 (función salud), 2020.

\begin{tabular}{|c|c|c|}
\hline \multicolumn{2}{|c|}{ Producto/actividad } & \multirow{2}{*}{$\begin{array}{l}\text { Finalidad } \\
\text { de desastres }\end{array}$} \\
\hline Producto 1 & 3000737. Estudios para la estimación del ries & \\
\hline Actividad 1.1 & $\begin{array}{l}\text { 5005572. Desarrollo de investigación aplicada } \\
\text { para la gestión del riesgo de desastres. }\end{array}$ & $\begin{array}{l}\text { 160788. Desarrollo de investigación aplicada } \\
\text { para la gestión del riesgo de desastres. }\end{array}$ \\
\hline Producto 2 & \multicolumn{2}{|c|}{$\begin{array}{l}\text { 3000738.Personas con formación y conocimiento en gestión del riesgo de desastres y adapta- } \\
\text { ción al cambio climático }\end{array}$} \\
\hline Actividad 2.1 & $\begin{array}{l}\text { 5005580. Formación y capacitación en materia } \\
\text { de gestión de riesgo de desastres y adaptación al } \\
\text { cambio climático }\end{array}$ & $\begin{array}{l}\text { 160796. Formación y capacitación en materia de } \\
\text { gestión de riesgo de desastres y adaptación al } \\
\text { cambio climático }\end{array}$ \\
\hline Producto 3 & \multicolumn{2}{|c|}{ 3000740.Servicios públicos seguros ante emergencias y desastres } \\
\hline Actividad 3.1 & $\begin{array}{l}\text { 5005570. Desarrollo de estudios de vulnerabili- } \\
\text { dad y riesgo en servicios públicos }\end{array}$ & $\begin{array}{l}\text { 160786. Desarrollo de estudios de vulnerabili- } \\
\text { dad y riesgo en servicios públicos }\end{array}$ \\
\hline Actividad 3.2 & $\begin{array}{l}\text { 5005585. Seguridad físico funcional de servicios } \\
\text { públicos }\end{array}$ & $\begin{array}{l}\text { 160801. Seguridad físico funcional de servicios } \\
\text { públicos }\end{array}$ \\
\hline Producto 4 & \multicolumn{2}{|c|}{ 3000739.Poblacion con prácticas seguras para la resiliencia } \\
\hline Actividad 4.1 & $\begin{array}{l}\text { 5005583.Organización y entrenamiento de } \\
\text { comunidades en habilidades frente al riesgo de } \\
\text { desastres }\end{array}$ & $\begin{array}{l}\text { 160799.0rganización y entrenamiento de } \\
\text { comunidades en habilidades frente al riesgo de } \\
\text { desastres }\end{array}$ \\
\hline Producto 5 & \multicolumn{2}{|c|}{$\begin{array}{l}\text { 3000734. Capacidad instalada para la preparación y respuesta frente a emergencias y } \\
\text { desastres }\end{array}$} \\
\hline Actividad 5.1 & $\begin{array}{l}\text { 5005561.implementación de brigadas para la } \\
\text { atención frente a emergencias y desastres }\end{array}$ & $\begin{array}{l}\text { 160777.Implementación de brigadas para la } \\
\text { atención frente a emergencias y desastres }\end{array}$ \\
\hline Actividad 5.2 & $\begin{array}{l}\text { 5005612. Desarrollo de los centros y espacios de } \\
\text { monitoreo de emergencias y desastres }\end{array}$ & $\begin{array}{l}\text { 160879. Desarrollo de los centros y espacios de } \\
\text { monitoreo de emergencias y desastres }\end{array}$ \\
\hline Actividad 5.3 & $\begin{array}{l}5005560 \text {. Desarrollo de simulacros en gestión } \\
\text { reactiva }\end{array}$ & $\begin{array}{l}160776 \text {. Desarrollo de simulacros en gestión } \\
\text { reactiva }\end{array}$ \\
\hline Actividad 5.4 & $\begin{array}{l}\text { 5005610. Administración y almacenamiento de } \\
\text { infraestructura móvil para la asistencia frente a } \\
\text { emergencias y desastres }\end{array}$ & $\begin{array}{l}\text { 160877. Administración y almacenamiento de } \\
\text { infraestructura móvil para la asistencia frente a } \\
\text { emergencias y desastres }\end{array}$ \\
\hline Producto 6 & 3000001. Acciones comunes & \\
\hline Actividad 6.1 & $\begin{array}{l}5004280 \text {. Desarrollo de instrumentos estraté- } \\
\text { gicos para la gestión del riesgo y la continuidad } \\
\text { operativa ante desastres }\end{array}$ & $\begin{array}{l}\text { 106777. Desarrollo de instrumentos estratégicos } \\
\text { para la gestión del riesgo y la continuidad opera- } \\
\text { tiva ante desastres }\end{array}$ \\
\hline Actividad 6.2 & $\begin{array}{l}\text { 5005609. Asistencia técnica y acompañamiento } \\
\text { en gestión del riesgo de desastres }\end{array}$ & $\begin{array}{l}\text { 160876. Asistencia técnica y acompañamiento en } \\
\text { gestión del riesgo de desastres }\end{array}$ \\
\hline Actividad 6.3 & $\begin{array}{l}\text { 5004279. Monitoreo, supervisión y evaluación } \\
\text { de productos y actividades en gestión de riesgo } \\
\text { de desastres }\end{array}$ & $\begin{array}{l}\text { 106813. Monitoreo, supervisión y evaluación de } \\
\text { productos y actividades en gestión de riesgo de } \\
\text { desastres }\end{array}$ \\
\hline
\end{tabular}


Tabla Nº3: Continuación...

\begin{tabular}{ll}
\hline Producto/actividad & Finalidad \\
\hline Actividad 6.4 5006144. Atención de actividades de emergencia & 0180105. Movilización y atención de brigadas \\
& 0180111. Monitoreo, evaluación y control de daños \\
& 0212133. Atención de daños a la salud de las \\
personas & \\
& 0212134. Control de brotes y epidemias \\
0212135. Tratamiento de agua para el consumo & humano \\
0212138. Atención de servicios esenciales frente & alluvias e inundaciones \\
0229090. Transporte e instalación de infraes- \\
tructura móvil, puentes modulares y albergues \\
0229091. Acciones de comunicación social \\
0229131. Atención de servicios esenciales frente \\
a emergencias y desastres
\end{tabular}

Fuente: Ministerio de Economía y Finanzas, 2020.

El análisis presupuestal consiste en el examen y explicación de los gastos e ingresos presupuestarios. El uso de indicadores presupuestarios (ratios) puede ayudar a mejorar la comprensión de cuestiones como el nivel de ejecución de los presupuestos de gastos e ingresos o la estructura del presupuesto. En el sector público, el objetivo principal del análisis presupuestal es controlar los gastos y predecir las necesidades presupuestarias futuras, proporcionando así a los tomadores de decisiones la información que necesitan para preparar el presupuesto para el próximo período fiscal (Balaguer, 2016).

El Consejo de Normas Internacionales de Contabilidad del Sector Público (IPSASB) 2014 define una serie de conceptos básicos relacionados con el presupuesto, como: (a) presupuesto aprobado; (b) presupuesto original; y (c) presupuesto final. El presupuesto aprobado significa la autorización de gastos sustentas en la ley, proyectos de ley de asignaciones, ordenanzas gubernamentales y otras decisiones vinculadas con los ingresos o recibos anticipados para el tiempo anual de presupuesto. El presupuesto original es el presupuesto inicial aprobado para el período presupuestario. El presupuesto final es el presupuesto original, ajustado para todas las reservas, montos transferidos, transferencias, asignaciones, asignaciones suplementarias y otros cambios de autoridad legislativa o similar autorizada aplicable al período presupuestario (Balaguer, 2016). 
El presupuesto se divide en presupuestos de ingresos y gastos. El presupuesto de ingresos se puede dividir en dos clasificaciones: administrativa y económica. El presupuesto de gastos puede tener tres clasificaciones: administrativa, económica y funcional. Además de estos tres sistemas de clasificación, algunos países utilizan sistemas adicionales para proporcionar información complementaria (Jacobs et al. 2009): (a) categorías por localización geográfica de la entidad administrativa, el contribuyente o el receptor de transferencias y subsidios gubernamentales; (b) categorías de los subsidios gubernamentales y beneficiarios de transferencias; (c) categorías de fuentes de financiamiento y contrapartida usados para el registro de donaciones externas y préstamos (Balaguer, 2016).

Para realizar un análisis presupuestal hay que considerar los indicadores de presupuesto. Existen algunos indicadores que pueden obtenerse de la información contenida en el presupuesto (Brusca y Condor, 2001; Carcaba, 2003; Balaguer, 2016).

Los indicadores de ejecución presupuestaria analizan en qué medida las previsiones y las asignaciones finales han llevado a la liquidación de las cuentas por cobrar y al reconocimiento de las obligaciones de pago, la facilidad con que estas cuentas por cobrar pueden convertirse en efectivo o la tasa a la que se realizan los pagos. Se pueden estudiar siguiendo la clasificación presupuestaria económica y / o funcional. Algunos de estos indicadores son:

(a) Ejecución de gastos: gastos totales (ejecutados) / estimaciones finales de gastos totales. Esta relación refleja la proporción de apropiaciones aprobadas en el período que han llevado al reconocimiento de obligaciones presupuestarias. Para un análisis más profundo, es útil desglosar la tasa de ejecución presupuestaria en sus componentes, como gasto corriente y gasto de capital y, para mayor detalle, en niveles como gasto en personal, bienes y servicios, etc. El indicador proporciona información sobre el presupuesto ejecutado en comparación con el presupuesto aprobado y, por lo tanto, es una referencia útil para evaluar los presupuestos futuros. Otra posibilidad es analizar el nivel de ejecución del presupuesto de gastos a la luz de su clasificación funcional; este análisis revelaría el alcance de la ejecución en los presupuestos aprobados para defensa, salud o educación, por ejemplo. 
(b) Ejecución de ingresos: ingresos totales (ejecutados) / previsiones finales de ingresos totales. Esta relación refleja la medida en que los ingresos presupuestarios aprobados (previstos) dan lugar a ingresos presupuestarios netos, es decir, el grado en que se ejecutaron. Partiendo de la clasificación económica del presupuesto, al igual que con el indicador anterior, también sería interesante descomponer la tasa de ejecución de los presupuestos de ingresos entre ingresos corrientes e ingresos de capital e incluso con un mayor nivel de detalle, como impuestos, transferencias de otros subsectores gubernamentales, etc.

(c) Ejecución de pagos: pagos totales / gastos totales ejecutados (obligaciones netas reconocidas). Este índice muestra los atrasos en el pago de los gastos, es decir, la proporción de obligaciones reconocidas en el período que fueron pagadas al final de ese período en relación al total de obligaciones reconocidas.

(d) Ejecución de cuentas por cobrar: cobros netos / ingresos totales ejecutados (ingresos netos reconocidos). Este índice muestra el porcentaje referido a las cuentas por cobrar obtenidas en el período sobre los ingresos netos reconocidos; por lo tanto, mide la capacidad de una entidad para convertir en sumas de efectivo que deben ser cobradas (Balaguer, 2016).

Los indicadores de la estructura presupuestaria son: (a) el porcentaje de gasto corriente: gasto corriente ejecutado / gasto total ejecutado (obligaciones netas reconocidas); esta razón mide el porcentaje del gasto total representado por el gasto corriente (salarios, bienes y servicios, transferencias corrientes y pagos de intereses); (b) el porcentaje de gastos de capital: gastos de capital ejecutados / gastos totales ejecutados (obligaciones netas reconocidas); esta razón refleja la fortaleza de inversión de la entidad. Establece la proporción del gasto presupuestario total representado por las operaciones de capital en el período; (c) la capacidad fiscal: capacidad fiscal propia (ingresos fiscales) / ingresos totales; esto refleja la proporción de los ingresos presupuestarios totales recaudados durante el período por los ingresos presupuestarios por impuestos (Balaguer, 2016).

El pliego presupuestal es la entidad que ejercen autoridad sobre las unidades ejecutoras; en este caso el pliego presupuestal es el MINSA para la unidad ejecutora, que es la DIRIS Lima Norte. 
Una unidad ejecutora puede hacer transferencias de una unidad a otra, esto es una transferencia externa, contando con la previa autorización del MINSA, que es pliego presupuestal; como también puede quitar de una unidad ejecutora con respecto a otra. Internamente, en una misma unidad ejecutora, como hay varios programas presupuestales, puede haber una priorización, en la medida que haya cumplimiento de alguna meta física, quitar dinero de un programa y colocarlo en otro, para que este puede tener un mejor desarrollo; se reitera que la condición es que haya cumplimiento de la meta física.

El artículo 5 de la Ley 28411 (Perú) muestra lo que es un pliego presupuestal en los siguientes términos: "5.1 Constituye Entidad Pública, en adelante Entidad, única y exclusivamente para los efectos de la Ley General, todo organismo con personería jurídica comprendido en los niveles de Gobierno Nacional, Gobierno Regional y Gobierno Local, incluidos sus respectivos Organismos Públicos Descentralizados y empresas, creados o por crearse; los Fondos, sean de derecho público o privado cuando este último reciba transferencias de fondos públicos; las empresas en las que el Estado ejerza el control accionario; y los Organismos Constitucionalmente Autónomos. 5.2 Constituyen pliegos presupuestarios las Entidades Públicas a las que se le aprueba un crédito presupuestario en la Ley de Presupuesto del Sector Público. 5.3 Los pliegos presupuestarios se crean o suprimen mediante Ley" (Ministerio de Economía y Finanzas, 2004).

El presupuesto del PP 068 tiene dos maneras de ser conformado: (a) al inicio del año que viene a ser el presupuesto inicial de apertura, es el presupuesto que el Estado asigna por aprobación del presupuesto general; un monto específico con el cual comenzar en el año., el cual proviene del Tesoro Público. Sin embargo, en el transcurso del año, puede ser que surjan nuevas necesidades, las cuales deben ser reflejadas en el presupuesto, es decir, añadidas a modo de transferencias. Esto hace que el presupuesto se vea modificado, incrementándose. A este presupuesto se le denomina ya (b) presupuesto inicial modificado. No obstante que lo normal es el incremento de gastos en el presupuesto, puede haber transferencias internas dentro de la unidad ejecutora o alguna entidad superior quite gastos de presupuesto. Debido a esta situación, por tanto, el presupuesto inicial de apertura puede verse mermado y bajar. Sobre el PIM es que se debe hacer el análisis presupuestal, en términos de lo que se ejecutó, ya que es el real presupuesto que se dispuso. 
Para el análisis presupuestal se debe considerar que los resultados de un programa presupuestal deben ser expresado en metas presupuestales y metas físicas y debe haber coherencia entrambas, es decir, deben guardar una perfecta relación, la cual asegura que el presupuesto se ha cumplido a cabalidad. En caso que ambas metas no guarden relación, se puede concluir que, en este caso, la DIRIS Lima Norte no establece correctamente la gestión del programa presupuestal.

Por tanto, el objetivo de esta investigación científica fue determinar la relación de las metas presupuestales con las metas físicas del PP 068 DIRIS Lima Norte, 2019.

\section{Metodología}

Esta investigación se basó en el paradigma o enfoque cuantitativo; tipo básico; diseño no experimental; corte longitudinal; alcance correlacional. La población estuvo conformada por todos los ítems mediante los cuales se dividió el presupuesto inicial modificado (PIM), a modo de una muestra censal. Los ítems a los que se hace referencia son los considerados como productos y actividades de la estructura funcional programática 2019 considerados por la DIRIS Lima Norte, expuesto en la tabla $\mathrm{N}^{\circ} 04$, los cuales se constituyen de cinco productos, una acción común y 12 actividades.

La información de interés: metas físicas y presupuestales, programadas y ejecutadas son de acceso público en el Portal de Transparencia Económica del Ministerio de Economía y Finanzas del Perú, denominado "Seguimiento de la Ejecución Presupuestal: Consulta amigable". Esta consideración de acceso público de la información brinda la validez ética para el uso de la información y, en tanto, responde a un análisis científico cumple con los estándares metodológicos. Para ello se usó el método hipotético-deductivo; el análisis documental, como técnica; y la hoja de cálculo, como instrumento.

Como parte del análisis estadístico se utilizará la prueba de Shapiro-Wilk para contrastar la normalidad del conjunto de datos a relacionar, dado que se considera uno de los test más potentes para el contraste de normalidad. Según el resultado obtenido se ampliará el análisis con la prueba de los rangos con signo de Wilcoxon, prueba no paramétrica para 
comparar el rango medio de dos muestras relacionadas y determinar si existen diferencias entre ellas, si la diferencia entre ellas se debe al azar o no (en este último caso, que la diferencia sea estadísticamente significativa).

Tabla $\mathrm{N}^{\circ} 04$

Estructura Funcional Programática PP 068 DIRIS Lima Norte, 2019.

\begin{tabular}{|c|c|}
\hline Productos & Actividades \\
\hline \multirow{3}{*}{ 3000001: Acciones comunes } & $\begin{array}{l}\text { 5004279: Monitoreo, supervisión y evaluación de productos y } \\
\text { actividades en gestión de riesgo de desastres (GRD) }\end{array}$ \\
\hline & 5004280: Desarrollo de instrumentos estratégicos para la GRD \\
\hline & 5005609: Asistencia técnica y acompañamiento en GRD \\
\hline \multirow{4}{*}{$\begin{array}{l}\text { 3000734: Capacidad instalada para la pre- } \\
\text { paración y respuesta frente a emergencias y } \\
\text { desastres }\end{array}$} & 5005560: Desarrollo de simulacros en gestión reactiva \\
\hline & $\begin{array}{l}\text { 5005561: Implementación de brigadas para la atención frente a } \\
\text { emergencias y desastres (E y D) }\end{array}$ \\
\hline & $\begin{array}{l}\text { 5005610: Administración y almacenamiento de infraestructura } \\
\text { móvil para la asistencia frente a E y D. }\end{array}$ \\
\hline & $\begin{array}{l}\text { 5005612: Desarrollo de los centros y espacios de monitoreo de } \\
\text { emergencias y desastres }\end{array}$ \\
\hline $\begin{array}{l}\text { 3000737: Estudios para la estimación del riesgo } \\
\text { de desastres }\end{array}$ & $\begin{array}{l}\text { 5005570: Desarrollo de estudios de vulnerabilidad y riesgo en } \\
\text { servicios públicos }\end{array}$ \\
\hline $\begin{array}{l}\text { 3000738: Personas con formación y conoci- } \\
\text { miento en GRD y adaptación al cambio climático }\end{array}$ & $\begin{array}{l}\text { 5005580: Formación y capacitación en materia de GRD y adapta- } \\
\text { ción al cambio climático }\end{array}$ \\
\hline $\begin{array}{l}\text { 3000739: Población con prácticas seguras para } \\
\text { la resiliencia }\end{array}$ & $\begin{array}{l}\text { 5005583: Organización y entrenamiento de comunidades en } \\
\text { habilidades frente al riesgo de desastres }\end{array}$ \\
\hline \multirow{2}{*}{ 3000740: Servicios públicos seguros ante E y D } & 5005584: Seguridad estructural de servicios públicos \\
\hline & 5005585: Seguridad físico funcional de servicios públicos \\
\hline
\end{tabular}

Fuente: Ministerio de Economía y Finanzas, 2019.

\section{Resultados y Discusión}

Tal como se expuso en el apartado de la metodología, se realizaron los análisis estadísticos a la información objeto de estudio, siendo los resultados de esta investigación los que se pueden evidenciar en la tabla $\mathrm{N}^{\circ} 05$, donde se expone la ejecución cuantificada en valores absolutos y porcentuales de las metas físicas y metas presupuestales, considérese para estas últimas metas que el presupuesto inicial de apertura (PIA) y el presupuesto inicial modificado (PIM) se expresan en soles (S/), según la estructura funcional programática del PP-068 para la Dirección de Redes Integradas de Salud Lima Norte, en el año 2019. 
Pensamiento Crítico Vol. 26. $\mathrm{N}^{\circ} 2$

Tabla $\mathbf{N}^{\circ} 05$

Metas físicas y presupuestales del PP 068 DIRIS Lima Norte, 2019.

\begin{tabular}{|c|c|c|c|c|c|c|c|c|}
\hline \multirow[b]{2}{*}{ Productos y actividades } & \multicolumn{5}{|c|}{ Metas presupuestales (S/) } & \multicolumn{3}{|c|}{ Metas físicas } \\
\hline & PIA & PIM & Ejec. & $\begin{array}{c}\text { Ejec. } \\
\%\end{array}$ & $\begin{array}{l}\text { Unidad } \\
\text { Med. }\end{array}$ & Prog. & Ejec. & $\begin{array}{c}\text { Ejec. } \\
\%\end{array}$ \\
\hline 3000001: Acciones comunes & 61,398 & 93,928 & 93,926 & 100 & Inf. Técnico & 67 & 75 & 115 \\
\hline $\begin{array}{l}\text { 5004279: Monitoreo, supervisión y } \\
\text { evaluación de productos y actividades en } \\
\text { gestión de riesgo de desastres (GRD) }\end{array}$ & 39,122 & 55,322 & 55,321 & 100 & Inf. Técnico & 56 & 61 & 109 \\
\hline $\begin{array}{l}\text { 5004280: Desarrollo de instr. Estratégicos } \\
\text { para la GRD }\end{array}$ & 5,232 & 1,762 & 1,762 & 100 & Inf. Técnico & 8 & 12 & 150 \\
\hline $\begin{array}{l}\text { 5005609: Asistencia técnica y acompaña- } \\
\text { miento en GRD }\end{array}$ & 17,044 & 36,844 & 36,843 & 100 & Inf. Técnico & 3 & 4 & 133 \\
\hline $\begin{array}{l}\text { 3000734: Capacidad instalada para la pre- } \\
\text { paración y respuesta frente a emergencias } \\
\text { y desastres }\end{array}$ & 39,101 & 55,827 & 52,509 & 94.1 & & & & \\
\hline $\begin{array}{l}\text { 5005560: Desarrollo de simulacros en } \\
\text { gestión reactiva }\end{array}$ & 2,887 & 2,887 & 2,086 & 72.3 & Reporte & 297 & 298 & 100 \\
\hline $\begin{array}{l}\text { 5005561: Implementación de brigadas } \\
\text { para la atención frente a emergencias y } \\
\text { desastres (E y D) }\end{array}$ & 12,700 & 28,511 & 28,510 & 100 & Brigada & 12 & 12 & 100 \\
\hline $\begin{array}{l}\text { 5005610: Administración y almacena- } \\
\text { miento de infraestructura móvil para } \\
\text { asistencia frente a E y D. }\end{array}$ & 6,175 & 7,090 & 4,574 & 64.5 & $\begin{array}{l}\text { Infraestruc- } \\
\text { tura móvil }\end{array}$ & 6 & 4 & 67 \\
\hline $\begin{array}{l}\text { 5005612: Desarrollo de los centros y } \\
\text { espacios de monitoreo de emergencias y } \\
\text { desastres }\end{array}$ & 17,339 & 17,339 & 17,339 & 100 & Reporte & 12 & 12 & 100 \\
\hline $\begin{array}{l}\text { 3000737: Estudios para la estimación del } \\
\text { riesgo de desastres }\end{array}$ & 27,678 & 58,217 & 58,216 & 100 & Doc. Técnico & 62 & 62 & 100 \\
\hline $\begin{array}{l}\text { 5005570: Desarrollo de estudios de vulne- } \\
\text { rabilidad y riesgo en servicios públicos }\end{array}$ & 27,678 & 58,217 & 58,216 & 100 & $\begin{array}{l}\text { Documento } \\
\text { técnico }\end{array}$ & 62 & 62 & 100 \\
\hline $\begin{array}{l}\text { 3000738: Personas con formación y cono- } \\
\text { cimiento en GRD y adaptación al cambio } \\
\text { climático }\end{array}$ & 368,834 & 338,293 & 337,419 & 99.7 & Persona & 377 & 417 & 111 \\
\hline $\begin{array}{l}\text { 5005580: Formación y capacitación en } \\
\text { materia de GRD y adaptación al cambio } \\
\text { climático }\end{array}$ & 368,834 & 338,293 & 337,419 & 99.7 & Persona & 377 & 417 & 111 \\
\hline $\begin{array}{l}\text { 3000739: Población con prácticas seguras } \\
\text { para la resiliencia }\end{array}$ & 27,601 & 35,401 & 34,096 & 96.3 & Persona & 337 & 345 & 102 \\
\hline $\begin{array}{l}\text { 5005583: Organización y entrenamiento } \\
\text { de comunidades en habilidades frente } \\
\text { al RD }\end{array}$ & 27,601 & 35,401 & 34,096 & 96.3 & Persona & 337 & 345 & 102 \\
\hline $\begin{array}{l}\text { 3000740: Servicios públicos seguros ante } \\
\text { Ey D }\end{array}$ & 41,187 & $2,551,166$ & $2,258,640$ & 88.5 & Intervención & 31 & 30 & 96.7 \\
\hline $\begin{array}{l}\text { 5005584: Seguridad estructural de servi- } \\
\text { cios públicos }\end{array}$ & 37,369 & 37,369 & 32,675 & 87.4 & Intervención & 1 & 0 & 0 \\
\hline $\begin{array}{l}\text { 5005585: Seguridad físico funcional de } \\
\text { servicios públicos }\end{array}$ & 3,818 & $2,513,797$ & $2,225,965$ & 88.6 & Intervención & 30 & 30 & 100 \\
\hline
\end{tabular}

Fuente: Ministerio de Economía y Finanzas, 2019. 
En la tabla $\mathrm{N}^{\circ} 06$ se puede observar la prueba de normalidad de Shapiro-Wilk, que a un nivel de significancia del $5 \%$ se obtiene un p-valor $<0,05$. Siendo la hipótesis nula que los datos están distribuidos normalmente y el p-valor es menor al nivel de significancia entonces la hipótesis nula es rechazada, es decir, se puede afirmar que los datos no describen una curva normal, siendo necesario ampliar el análisis con el estadígrafo de Wilcoxon.

Tabla $\mathbf{N}^{\circ} 06$

Pruebas de normalidad.

\begin{tabular}{llll}
\hline & Shapiro-Wilk & & \\
& Estadístico & Gl & Sig. \\
\hline Metas presupuestales &, 696 & 12 &, 001 \\
Metas físicas &, 791 & 12 &, 007 \\
\hline
\end{tabular}

a. Corrección de la significación de Lilliefors

Fuente: Elaboración propia

En la tabla $\mathrm{N}^{\circ} 07$, utilizando la en la prueba de Wilcoxon se puede observar que a un nivel se significancia de 5\% se obtuvo un p-valor $>0,05$. Por lo que, se afirma la hipótesis nula que establece que la ejecución de las metas presupuestales no se relaciona con la ejecución de las metas físicas del PP 068 DIRIS Lima Norte, 2019.

\section{Tabla $\mathbf{N}^{\circ} 07$}

Prueba de Wilcoxon para las variables.

\begin{tabular}{ll}
\hline & Metas físicas - Metas presupuestales \\
\hline $\mathrm{Z}$ & $-1,599^{\mathrm{b}}$ \\
Sig. asintót. (bilateral) & 110 \\
\hline
\end{tabular}

Estadísticos de contraste: a. Prueba de los rangos con signo de Wilcoxon y b. Basado en los rangos negativos.

Fuente: Elaboración propia

Así mismo, esto se traduce en cómo las condiciones reales en el análisis presupuestal no muestran una relación entre la ejecución de las metas presupuestadas y las metas físicas de la DIRIS Lima Norte, mediante lo cual puede establecerse dos situaciones: (a) la baja ejecución presupuestal con alta ejecución de metas físicas; o (b) la alta ejecución presupuestal 
con baja ejecución de metas físicas. La coherencia en una buena gestión de un programa presupuestal se sustenta en dar sentido al binomio presupuesto-resultado físico, con lo cual se construyen los productos de entrega a la población objetivo. Los desvíos (la falta de relación) constituyen diferentes vicios que se cometen en las fases de gestión de un programa presupuestal, desde la planificación o programación, la organización, la ejecución y en el proceso de seguimiento (monitoreo y evaluación).

Esta conclusión general tiene aristas de análisis que no son sino la subsunción de las actividades que han sido implementadas de manera programada con un presupuesto asignado, es pues meritorio hacer una deliberación individual de las actividades para una mejor y mayor discusión.

La actividad "Monitoreo, supervisión y evaluación de productos y actividades en gestión de riesgo de desastres", en la DIRIS Lima Norte, comprendió 61 acciones de control, aun cuando se programaron 56 (115\%) con un presupuesto ejecutado al 100\% de S/93,926. Las acciones de control se desarrollaron en base a los instrumentos estratégicos para la GRD, por ejemplo, se monitorizó el Plan de Contingencia en Fiestas Patrias en el Hospital Nacional Cayetano Heredia, Hospital Carlos Lanfranco La Hoz, Hospital Nacional Sergio Bernales, Centro de Salud Tahuantinsuyo Bajo e Instituto Nacional de Salud Mental Honorio Delgado-Hideyo Noguchi y el Plan de Contingencia "Fiestas de Fin de Año" en las épocas de fin de año a los 11 Centros de salud maternos infantiles, entre otros. Esto significa que habiendo un 15\% más de logro de metas físicas, el costo para el desarrollo de esta actividad es menor al estimado en la programación inicial.

La actividad "Desarrollo de instrumentos estratégicos para la GRD” comprendió el desarrollo de 12 planes para la GRD; principalmente, planes de contingencias, aun cuando se programaron 8 (150\% de ejecución), con una ejecución presupuestal del 100\%. Estos planes permitieron el despliegue de las acciones en el año y debido a que eran recurrentes las contingencias en la DIRIS Lima norte, vinculadas a fechas festivas y riesgos previamente identificados, se debió mejorar la programación de la meta física para no incurrir en la misma incoherencia físico-presupuestal

La actividad "Asistencia técnica y acompañamiento en GRD”, al igual que la actividad previa requirieron una mejor programación de su meta física, debido a que la ejecución del $133 \%$ no fue coherente con el 100\% 
de ejecución del presupuesto. Así mismo, en las operaciones el criterio de programación determinaba que se realice un informe por mes, es decir, la meta debió ser de 12 informes técnicos.

La actividad "Desarrollo de simulacros en gestión reactiva" evidenció una correcta ejecución de meta física (100\%); sin embargo, una ejecución de presupuesto deficiente (72.3\%). Considerando la definición operacional de esta actividad, la cantidad de 104 establecimientos de salud y la indicación de tres simulacros nacionales que se debieron programar con 312 reportes de simulacros. Con lo cual se evidenció que, a pesar de haber cumplido una ejecución al 100\% en la meta física, su programación fue inadecuada.

La actividad "Implementación de brigadas para la atención frente a emergencias y desastres" tuvo una ejecución física y presupuestal coherente y satisfactoria del $100 \%$.

La actividad "Administración y almacenamiento de infraestructura móvil para la asistencia frente a emergencias y desastres" tuvo un ejecución física y presupuestal coherente, aunque insatisfactoria debido a que ambos avances estuvieron en $67 \%$ y $64.5 \%$, respectivamente.

La actividad "Desarrollo de los centros y espacios de monitoreo de emergencias y desastres (EMED)" tuvo una ejecución física y presupuestal coherente y satisfactoria del 100\%. La DIRIS Lima Norte tiene su EMED con un funcionamiento de 24 horas diariamente. Los reportes consolidados evidenciaron que el promedio de emergencias mensual fue de 158, un total anual de 1901, con una distribución proporcional entre incendios, accidentes de tránsito y otros incidentes, siendo la fuente principal las emergencias reportadas por los bomberos.

La actividad "Desarrollo de estudios de vulnerabilidad y riesgo en servicios públicos" tuvo una ejecución física y presupuestal coherente y satisfactoria del 100\%. La DIRIS Lima Norte realizó 62 documentos técnicos de índices de seguridad de los establecimientos de salud del primer nivel de atención, donde ninguno fue categorizado como "A", el 39\% fue de categoría "B" y 61 \% fue de categoría "C". Siendo los EESS de categoría I-2 (Puestos de Salud) los más vulnerables, dado que el 68\% de ellos son de categoría "C". 
La actividad "Formación y capacitación en materia de GRD y adaptación al cambio climático" permitió capacitar a 417 personas de las 377 programadas (111\%) de los EESS en las siguientes temáticas: (a) sistema de comando de incidentes; (b) brigadas intervención inicial en emergencias y desastres; (c) espacios de monitoreo de emergencias y desastres; y (d) evaluación de daños y necesidades, con una ejecución presupuestal del 99.7\%.

La actividad "Organización y entrenamiento de comunidades en habilidades frente al riesgo de desastres” permitió capacitar a 345 personas de las 337 programadas (102\%), aunque con un enfoque de instituciones/ actores sociales de prioridad: Ejército Peruano del Fuerte General Belisario Suarez de Ancón, Colegio Libertador San Martín, Asociación Central de Promotoras De Salud (ACEPRODES) y Barrio Seguro Péndulo de Lomas de Carabayllo; la temática fue: (a) primeros auxilios y reanimación cardiopulmonar; (b) gestión de riesgo de desastres; y (c) plan familiar;, con una ejecución presupuestal del 96.3\%.

La actividad "Seguridad físico-funcional de servicios públicos" reportó 30 intervenciones; principalmente, en base a la Resolución Secretarial N²82-2019-MINSA (16/10/2019), mediante la cual se realizó una transferencia para financiar las actividades de mantenimiento de techos y coberturas de 26 establecimientos de salud para enfrentar precipitaciones pluviales, incorporadas en los Planes Multianuales de Mantenimiento de la Infraestructura y el Equipamiento en los Establecimientos de Salud - Lima Metropolitana 2019-2021, por la Resolución Ministerial № 8902019/MINSA. No obstante, el reporte del 100\% de cumplimiento de meta física, la ejecución presupuestal no fue coherente con ella, por haber sido del $88.6 \%$.

La actividad "Seguridad estructural de servicios públicos" se relacionó a la seguridad estructural de servicios públicos. Se programó una intervención; sin embargo, no obstante, a que hubo ejecución de presupuesto del $87.84 \%$ (S/ 32,675.00) en la actividad no se ejecutó la meta física, lo cual generó una incoherencia de ejecución físico-presupuestal, la cual requiere ser objeto de indagaciones a fin de evidenciar en qué se ejecutó dicho presupuesto. 


\section{Conclusiones y Recomendaciones}

En conclusión, en el 2019, el Programa Presupuestal 068 en la DIRIS LN inició el año con un PIA de S/ 565,799.00 soles, y culminó con un PIM de S/ 3132832.00 soles. En promedio ejecutó el 90.5\% del PIM y un cumplimiento del $98 \%$ de metas físicas, sin embargo, las metas presupuestales no se relacionaron con las metas físicas del PP 068 DIRIS Lima Norte.

$\mathrm{Al}$ constatar que las metas presupuestales no se relacionan con las metas físicas del PP 068 DIRIS Lima Norte durante la ejecución en el año 2019, se recomienda que se consideren los alcances de la evaluación de la implementación del PP 068 del año 2019, para una mejor programación de metas físicas y presupuestales en años siguientes; así como el monitoreo y evaluación constante para el logro de una armonía en la ejecución del binomio meta presupuestal - meta física.

\section{Referencias Bibliográficas}

Balaguer, M. (2016). Budget Analysis. En: Encyclopedia of Public Administration, Public Policy, and Governance, A. Farazmand (ed.), Global DOI 10.1007/9783-319-31816-5_2260-1

Brusca, M. y Condor, V. (2001) El análisis financiero en las administraciones locales. Span J Financ Account 30(108);475-504.

Carcaba, A. (2003). Análisis financiero de las entidades locales mediante el uso de indicadores. Span J Financ Account 32(118);661-692.

Dirección General de Cambio Climático y Desertificación. (2018). Lineamientos para la incorporación de criterios sobre infraestructura natural y gestión del riesgo en un contexto de cambio climático, en el marco de Reconstrucción con Cambios [DS N017-2018-MINAM]. Lima: Ministerio del Ambiente. Disponible en: https://www.senace.gob.pe/wp-content/uploads/2019/03/Tema8-Lineamientos-incorporacion-de-criterios-sobre-infraestructura-naturaly-gestion-del-riesgo.pdf

DIRIS Lima Norte. (2020). Conócenos. Lima: Ministerio de Salud -MINSA. Recuperado de: http://www.dirislimanorte.gob.pe/conocenos/, el 29 de agosto de 2020.

El Peruano. (2011). Ley № 29664, Ley que crea el Sistema Nacional de Gestión del Riesgo de Desastres (SINAGERD). Lima. Recuperado de: https://busquedas. 
elperuano.pe/normaslegales/ley-que-crea-el-sistema-nacional-de-gestiondel-riesgo-de-de-ley-n-29664-605077-1/, el 29 de agosto de 2020

Gob.pe. (2019). Presupuesto por resultados. Lima: Gob.pe Plataforma Digital del Estado Peruano. Recuperado de: https://webcache.googleusercontent.com/ search?q=cache:949PZMcAx1MJ:https://www.gob.pe/843-ministerio-deeconomia-y-finanzas-presupuesto-por-resultados $+\& \mathrm{~cd}=1 \& \mathrm{hl}=\mathrm{es} \& \mathrm{ct}=\mathrm{clnk}$ $\& g l=p e$, el 20 de agosto de 2020.

INDECI. (s/f). Lineamientos para la respuesta. Proceso de la gestión del riesgo de desastres. Lima: Instituto Nacional de Defensa Civil del Perú. Disponible en: https://www.indeci.gob.pe/wp-content/uploads/2018/10/LINEAMIENTOS_PARA_LA_RESPUESTA_2.pdf

IPSASB. (2014). Handbook of international public sector accounting pronouncements, vol I and II. New York: International Federation of Accountants, IFAC.

Jacobs, D., Helis, J. y Bouley, D. (2009). Budget classifications, technical notes and manuals. Washington, DC: International Monetary Fund, Fiscal Affairs Department.

Kuroiwa, J. (2010). Alto a los desastres. Viviendas seguras y saludables para los peruanos con menores recursos. Lima: Umbral Ediciones S.A.C.

Ministerio de Economía y Finanzas. (2004). Ley General del Sistema Nacional de Presupuesto, Ley № 28411. Lima. Disponible en: https://www.mef.gob.pe/ es/por-instrumento/ley/5539-ley-n-28411/file

Ministerio de Economía y Finanzas. (2017). Programa presupuestal 068. Reducción de la vulnerabilidad y atención de emergencias por desastres. Disponible en: https://www.mef.gob.pe/contenidos/presu_publ/ppr/prog_presupuestal/articulados/prog_pptal_068_2017.pdf

Ministerio del Ambiente. (2011). Decreto Supremo que aprueba el Reglamento de la Ley № 29664, que crea el Sistema Nacional de Gestión del Riesgo de Desastres (SINAGERD) Decreto Supremo № 048-2011-PCM. Disponible en: http://www.minam.gob.pe/prevencion/wp-content/uploads/ sites/89/2014/10/2.-DS-048-2011-Reglamento-Ley-29664.pdf

Pontificia Universidad Católica del Perú. (2016). Desastres y fenómenos naturales en el Perú: Gestión y prevención ante los riesgos. Lima. Disponible en: https:// inte.pucp.edu.pe/wp-content/uploads/2016/11/IV-BIBLO_Programa.pdf

PREVAED. (2019). Diseño del programa presupuestal 068. Lima: Viceministerio de Gobernanza Territorial - Programa Presupuestal de Reducción de la Vulnerabilidad y Atención de Emergencias por Desastres. 
PREVAED. (2020). Contenidos mínimos del programa presupuestal. Lima: Presidencia del Consejo de Ministros - Programa Presupuestal de Reducción de la Vulnerabilidad y Atención de Emergencias por Desastres. Disponible en: http://www.pcm.gob.pe/wp-content/uploads/2019/02/ContenidosM\%C3\%ADnimos-del-Programa-Presupuestal-068-2019-compressed.pdf

Programa Nuestras Ciudades. (2014). Sistema Nacional de Gestión del Riesgo de Desastres y los Mecanismos Financieros para la GRD. Disponible en: https:// docplayer.es/storage/78/78150264/1598765400/iugfA5rweNTunpebNUytQ/78150264.pdf

\section{Notas al final}

1 Artículo de Investigación Científica. La realidad de la Gestión del Riesgo de Desastres en el área de la salud tiene matices propios que deben ser analizados en los diferentes procesos aplicativos (estimación, prevención y reducción del riesgo, preparación, respuesta, rehabilitación y reconstrucción), siendo una línea de trabajo muy sensible en Lima, por su nivel de vulnerabilidad y en el lado Norte de ella, por la gran cantidad de personas que allí residen (2 909340 habitantes).

2 Médico Cirujano, Universidad Peruana Cayetano Heredia, Lima, Perú. Especialista en Gestión en Salud, Universidad Nacional Mayor de San Marcos, Lima, Perú. Egresado de Maestría de Gestión Económica de Riesgo de Desastres y Desarrollo Sostenible, Universidad Nacional Mayor de San Marcos en Lima, Perú. Asesor de Dirección General de la Dirección Regional de Salud de Ucayali. Teléfono: 994 729 378. Correo-e: gonguz.beto@gmail.com. 